\title{
Visible Light Induced Charge Retention and Photocatalysis with Hybrid CdSe-Au Nanodumbbells
}

RONNY COSTI, AARON E. SAUNDERS, EINAT ELMALEM, ASAF SALANT AND URI BANIN

Institute of Chemistry and the Center for Nanoscience and Nanotechnology, The Hebrew University of Jerusalem, Jerusalem 91904, Israel

*banin@chem.ch.huji.ac.il, phone: +972-2-6584515, fax: +972-2-6584148

\section{$\underline{\text { Supporting Information }}$}

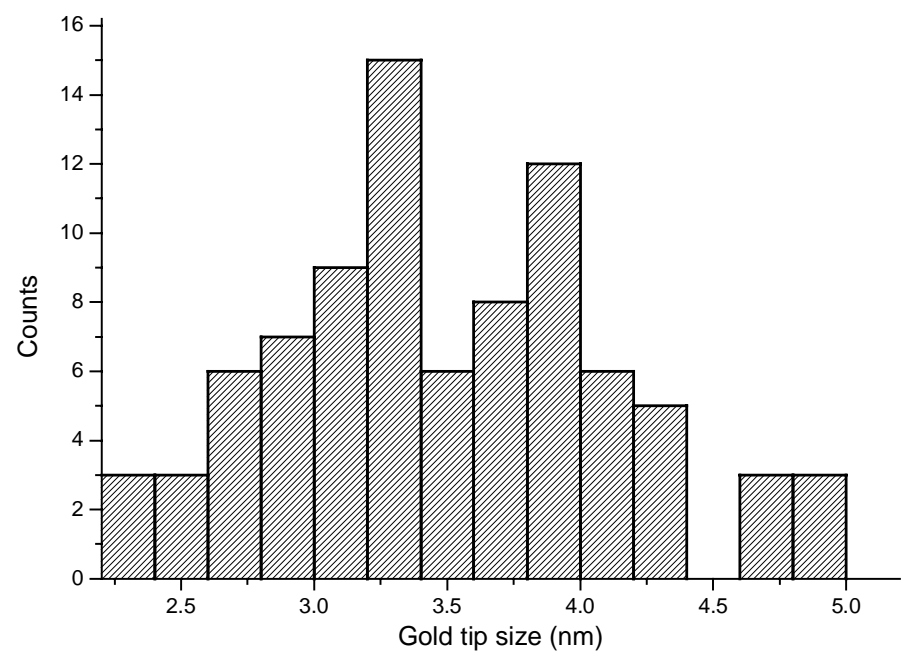

Supporting figure S1: Size distribution of gold tips on $\sim 22 \mathrm{x} 4 \mathrm{~nm}$ nanodumbbells. 


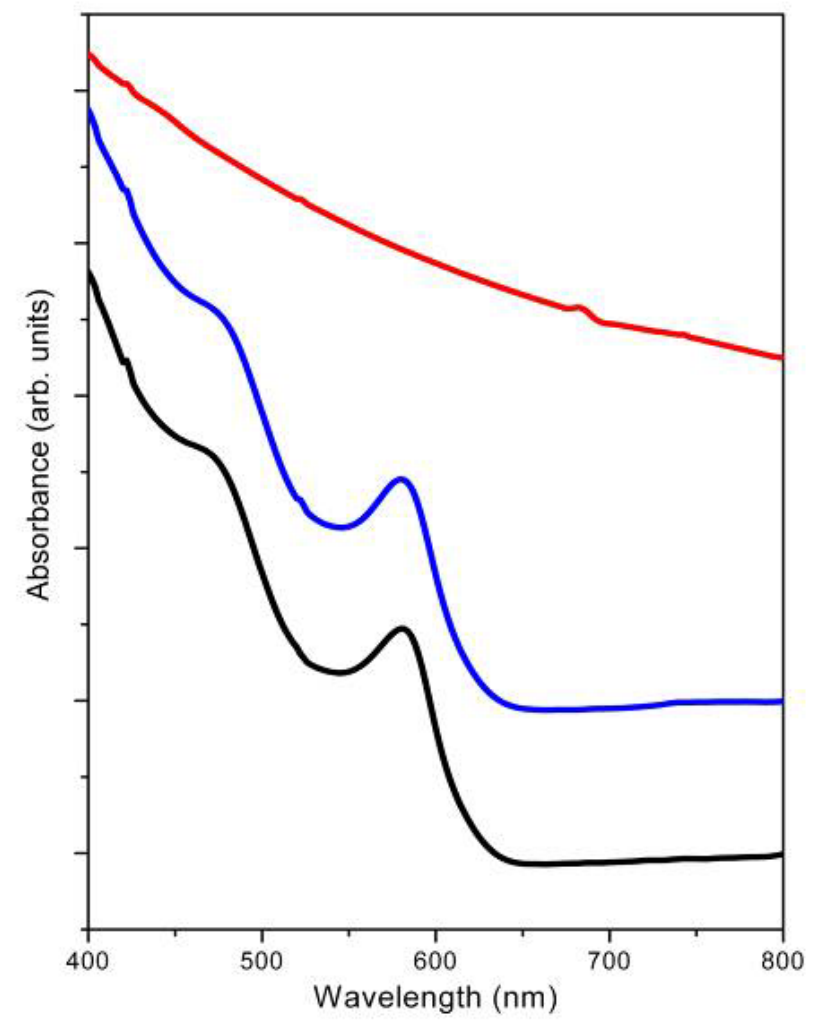

Supporting figure S2: Absorbance spectra of CdSe rods $(\sim 38 \times 4 \mathrm{~nm})$ in chloroform solution (black line) and the same nanorods in aqueous solution (blue line) demonstrating the excitonic peak is maintained, and after growing gold tips on them in aqueous solution (red line) where the exciton feature is washed out (spectra are shifted vertically for clarity). 


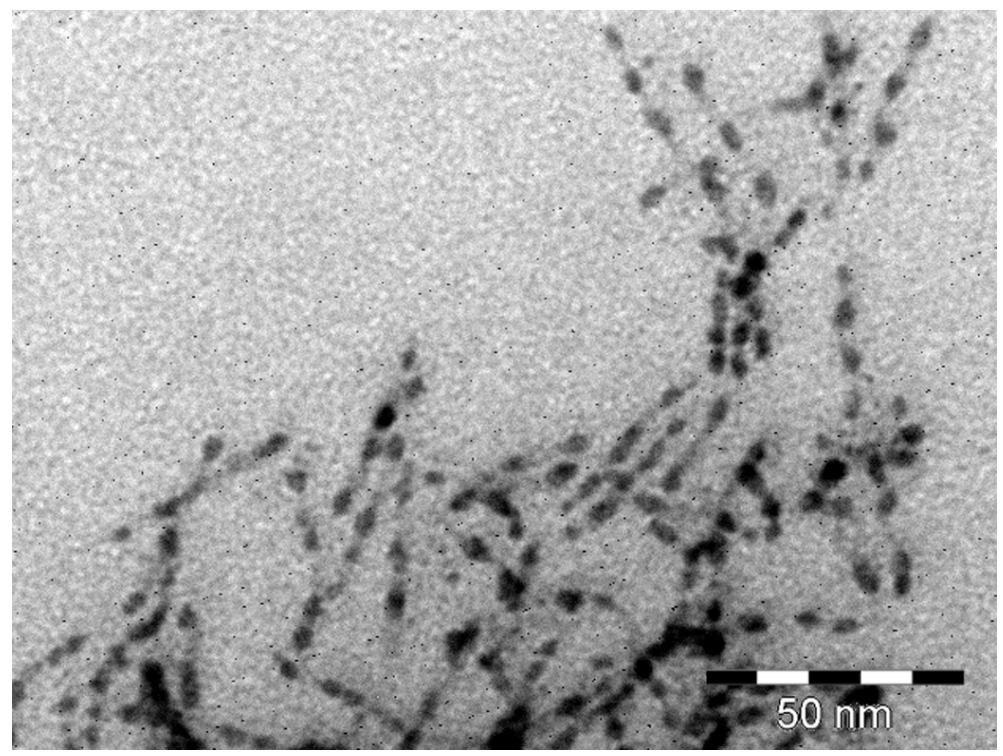

Supporting figure S3: TEM image of NDBs grown in total darkness conditions. Gold growth is seen even without light. It should be noted that the TEM grids used for this analysis were hydrophobic carbon coated grids, and as a result NDBs aggregated upon deposition and solvent evaporation. In Figure 1 in the article ionized grid was used, rendering it hydrophilic, and improving the sticking of NDBs from the aqueous solution preventing aggregation.

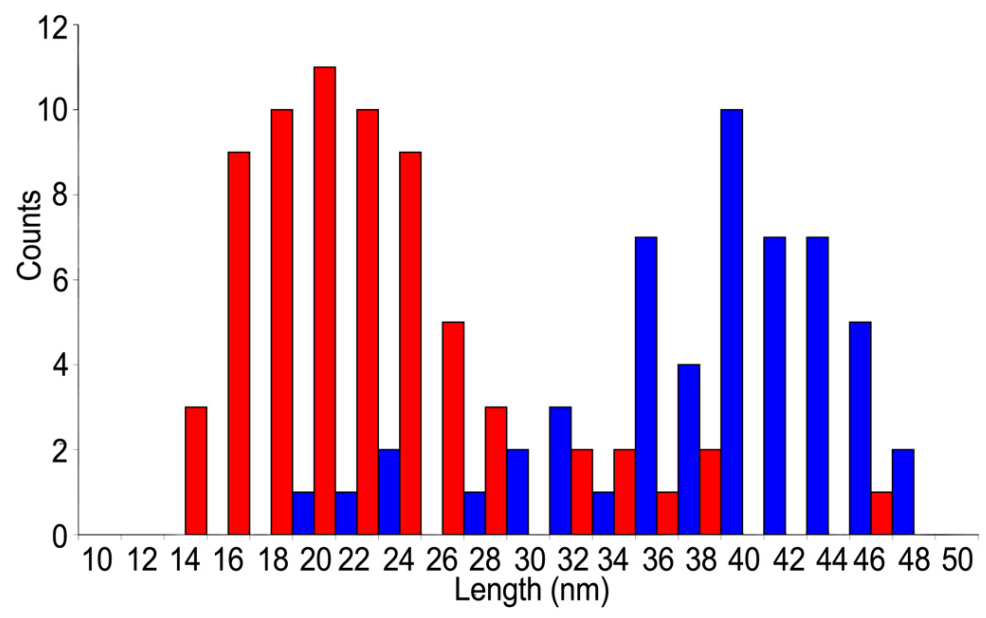

Supporting figure S4: Length distribution of CdSe nanorods templates in aqueous solution (Blue) and of CdSe-Au nanodumbbells from the same nanorods (Red) showing a distinct shortening of the rods length due to sacrificial etching of the CdSe upon the reduction of gold. 

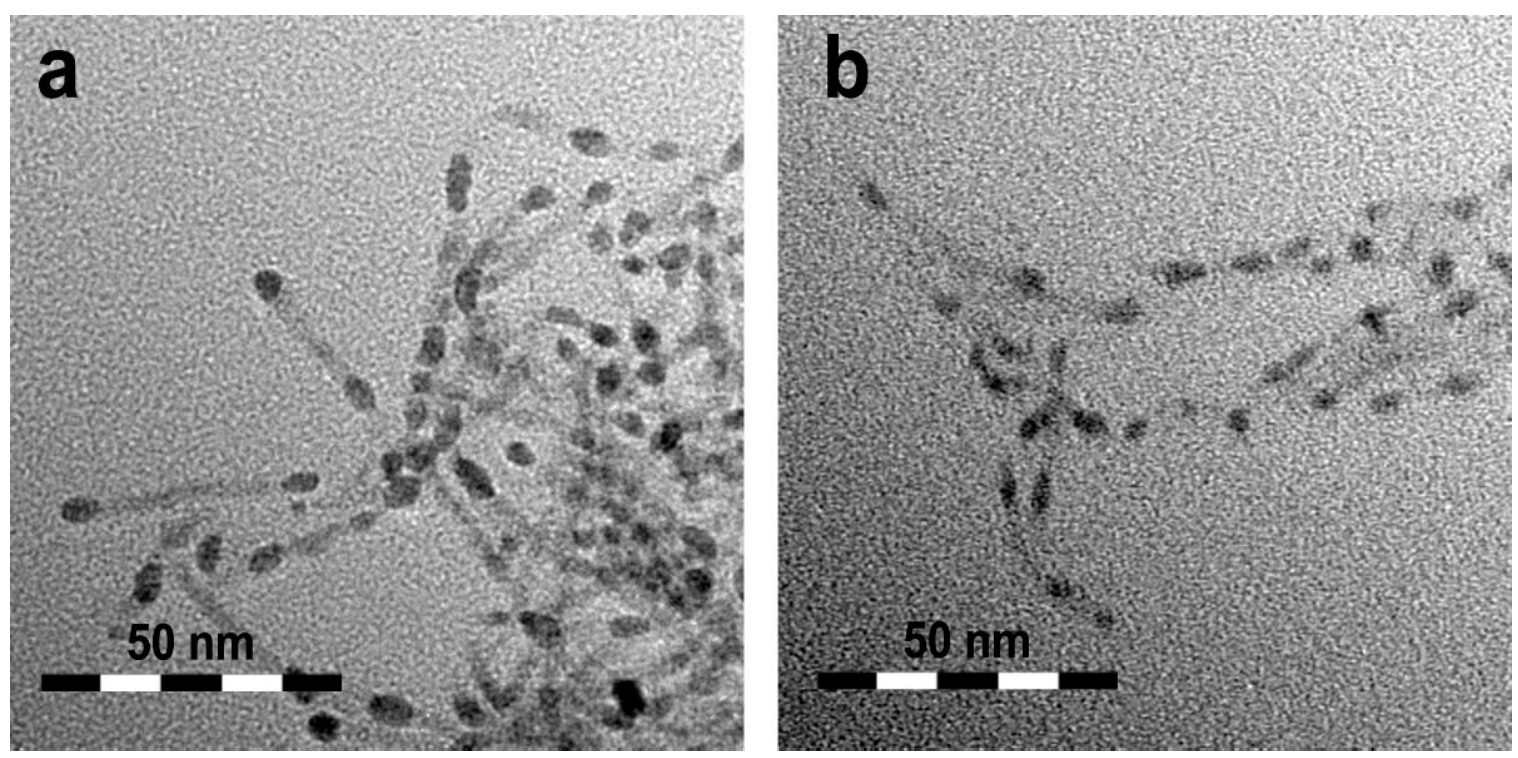

Supporting figure S5: TEM images of CdSe-Au nanodumbbells ( $40 \mathrm{X} 4 \mathrm{~nm}$ ) before (a) and after (b) 30 minutes of irradiation using 532nm laser, demonstrating a similar morphology of the particles despite the irradiation. The presence of a buffer solution causes aggregation on the TEM grid and a reduced contrast.
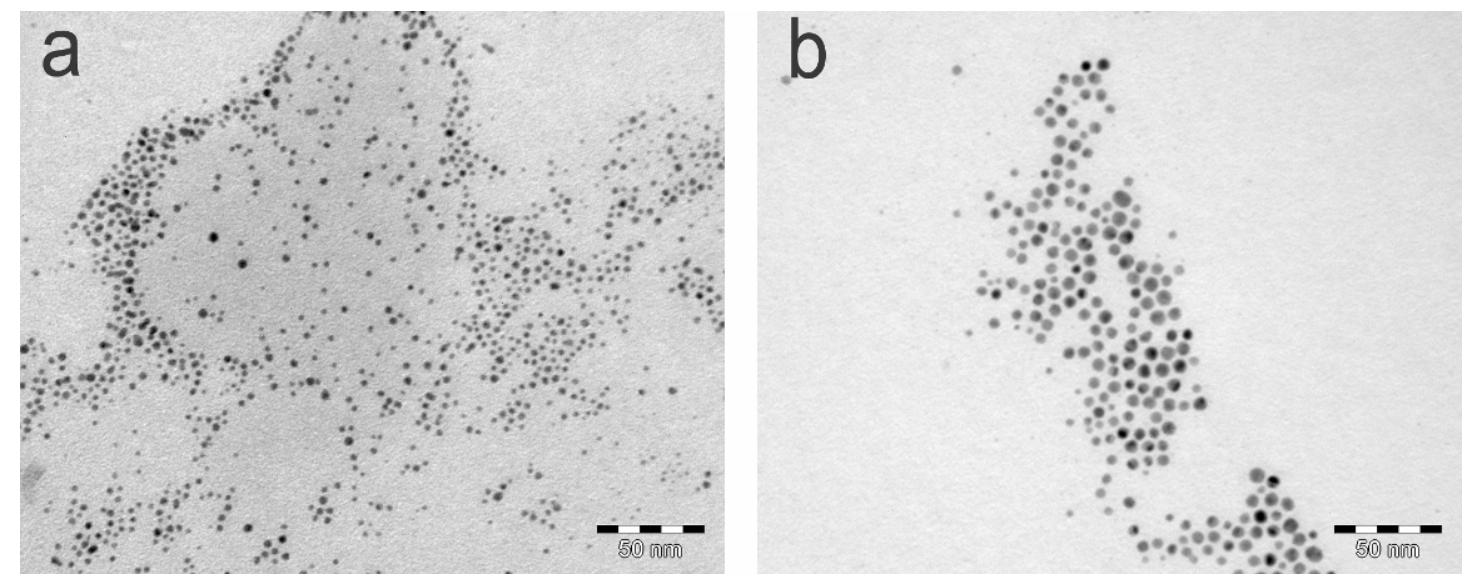

Supporting figure S6: TEM images of gold nanoparticles used for control experiments of simultaneous irradiation with MB. (a) $4( \pm 1.3) \mathrm{nm}$ particles and (b) $6( \pm 1) \mathrm{nm}$ particles. 